This is a postprint version of the following published document:

Sandonis, V., Soto, I., Calderón, M., Fernández, I., Vidal, I. (2015). CATMISS: context-aware transparent mobility for IMS services. Multimedia Tools and Applications, 74(13), 4789-4816

DOI: http://dx.doi.org/10.1007/s11042-013-1839-4

(C) Springer Science+Business Media New York 2014 


\title{
CATMISS: Context-Aware Transparent Mobility for IMS Services
}

\author{
Victor Sandonis, Ignacio Soto*, Maria Calderon, Ismael Fernandez, Ivan Vidal
}

Departamento de Ingeniería Telemática

Universidad Carlos III de Madrid

Avda. de la Universidad, 30

28911 Leganés - Madrid (Spain)

vsandoni@it.uc3m.es, isoto@it.uc3m.es, maria@it.uc3m.es, ismafc06@gmail.com, ividal@it.uc3m.es

\begin{abstract}
The IP Multimedia Subsystem (IMS) is considered as the common platform for providing a unified session control on top of various access network technologies for multimedia applications. At the same time, we are witnessing how wireless technologies become an integral part of our daily life, due to an increasing number of mobile multi-interface devices and the proliferation of wireless access networks. In this context the availability of transparent mobility management mechanisms is a key element to enhance user's quality of experience.

On the other hand, context-awareness is a vital ingredient to allow mobile devices to make appropriate and timely mobility decisions. The benefits of using diverse context information (e.g., about the user, the service and the network) to manage mobility are twofold. First, the richer information is available, the more likely an intelligent decision can be taken. Second, it allows the network to assist the terminal to take mobility decisions, even up to the point of automatically initiating a mobility procedure if according to the context and user preferences this improves the user's quality of experience.

In this paper we propose CATMISS, a solution to support context-aware mobility in IMS services. The solution is based on SIP signalling and makes mobility transparent for communication peers of mobile terminals. Two types of mobility are supported: a terminal changing of access network and a multimedia session being transferred from one terminal to another. The paper also describes the implementation of the solution and its deployment in an experimental platform. Experiments in this platform, with IPTV and videoconference services, are used to validate the proposal.
\end{abstract}

\section{Keywords}

Context-awareness, transparent mobility, IMS, SIP

\section{INTRODUCTION}

Several trends are pushing the evolution of communication networks nowadays. One of them is the adoption of Internet technologies, and in particular the IP protocol, as the cornerstone of operator networks. Multimedia services, such as VoIP or IPTV, are already being provided through IP-based operator networks. Real-time multimedia services require specific functionality such as informing participants of incoming communications and preparing the transport layer to offer an appropriate quality of experience to users. The IP Multimedia Subsystem (IMS [1]) is the framework that operators are adopting to provide this functionality. The key protocol in IMS is the Session Initiation Protocol (SIP [2], [3]), a signalling protocol to establish, modify, and terminate sessions between users with the adequate resources in the transport plane.

Another trend in the evolution of networks is the prominent role of mobility. Users have sophisticated terminals with multiple network interfaces and demand ubiquitous access to their services, even while on the move. To fulfil this requirement operators have to fit their networks with a combination of heterogeneous wireless access technologies that offer a good balance between costs, coverage, and available bandwidth. Moreover, to improve the service offered to their users and help in

\footnotetext{
${ }^{*}$ Corresponding author, Tel: +34 916245974; Fax: +34 916248749

This is the accepted version of the following article: "CATMISS: context-aware transparent mobility for IMS services. Victor Sandonis, Ignacio Soto, Maria Calderon, Ismael Fernandez, Ivan Vidal. Multimedia Tools and Applications, Vol. 74, No. 13, July 2015. Pages: 4789-4816. ISSN: 1380-7501", which has been published in final form at http://dx.doi.org/10.1007/s11042-013-1839-4.
} 
reducing infrastructure costs, operators can choose to also allow their users to obtain connectivity through access networks belonging to third parties (for example, other operators).

In this environment users will move and the point of attachment of their terminals to the network will change. A terminal that moves to a new point of attachment will typically need to change its IP address which will affect the open communications in the terminal. There are several proposals to deal with the problems caused by mobility in IP networks. Network-based IP mobility support solutions, such as GTP [4] or PMIPv6 [5], are designed to hide mobility from the terminal, which will not need to change its IP address. However, these solutions are restricted to some area: the points of attachment to the network that are covered by the corresponding functionality. For example, it cannot support movements to access networks of other operators. On the other hand, several client-based IP mobility support solutions have been developed by the IETF ${ }^{1}$, such as Mobile IPv4 [6] or Mobile IPv6 [7], but directly combining these solutions with IMS does not work [8], [9], [10], because they use two IP addresses: one permanent, seen by applications, that is used as identifier; and another one, that changes in each visited network, used as locator to route the traffic. In IMS, SIP is an application layer protocol and thus sees the permanent address. However, this protocol is used to control the reservation of resources for the communication sessions and that has to be done for the locator address that is the one used for routing in the network. This is a conflict that has not an easy solution without making IMS aware of the IP mobility solution, but modifying IMS is undesirable, so a different approach is needed to offer mobility in IMS networks.

Another type of mobility that is especially attractive for multimedia services is session mobility [11], the movement of a communication session from one terminal to another. This allows a user with several terminals to move sessions between them, for example moving an active communication session from a mobile terminal to a fixed terminal with better capabilities when available.

Unfortunately, all this functionality also comes at the cost of increasing the complexity. We have an environment with several services available, several access networks, several network conditions, several terminals, and so on. Therefore it is increasingly difficult for users to manually take decisions to operate in this environment, so a system that automates or helps with decisions is needed. On the other hand, to enrich their experience, decisions have to take into account context information about the users, the services, and the network.

In this paper we propose CATMISS, a context-aware transparent mobility support solution based on SIP for IMS services. This solution has been developed in the context of the UP-TO-US Celtic project ${ }^{2}$. CATMISS uses mobility procedures that are based on those defined in TRIM [12], a solution to provide transparent terminal mobility in IMS networks, but with significant differences that will be explained in section 2, in which we analyse the related work. CATMISS supports terminal and session mobility for regular IMS user equipment, just requiring the ability of the applications in the mobile terminal to deal with changes of IP address. CATMISS keeps terminal and session mobility completely transparent to the communication peers of the mobile terminal. The last feature of CATMISS is that its operations are managed with the help of a contextawareness server, which collects information about users, communications, terminals, access networks, and preferences, and allows enhancing decision making about terminal and session mobility. The strategy of our context awareness solution is to concentrate the operations in servers in the network, to be able to use complex algorithms, e.g. learning algorithms, applied to large quantities of information without overloading the terminals and the wireless accesses. A preliminary version of our work was presented in [13], where a simplified version of the context-aware system was presented, only outlining some examples of its expected usefulness, but without including a complete description of the system. In particular, aspects of the context-aware system that [13] does not cover are: the systematic description of the context information that is managed by the system, the types of mobility triggers generated, how the context information is collected, and the format used for the exchange of context information. Here we develop the context-awareness system covering the aforementioned aspects, integrating the context aware messages and triggers in the mobility procedures, and also including the described context aware system in our prototype for the evaluation.

The rest of the paper is organized as follows: section 2 gives an overview on the related work in mobility and contextawareness systems in IMS networks; section 3 describes the proposed solution for context-aware transparent SIP-based mobility for IMS services; section 4 presents an analysis of the proposed solution; and, finally, section 5 highlights the conclusions that result from our work.

\section{RELATED WORK}

There are several proposals that deal with mobility support in IMS. A first option is to use SIP [2], the core protocol in IMS, to provide that mobility support. SIP uses identifiers (Uniform Resource Identifiers, URIs) for users, and can keep track and

\footnotetext{
${ }^{1}$ Internet Engineering Task Force, http://www.ietf.org

${ }^{2}$ UP-TO-US Celtic project: http://www.celtic-initiative.org/Projects/Celtic-projects/Call7/UP-TO-US/uptous-default.asp
} 
inform other parties of the IP address of the user terminal, including when that address changes because of a movement. This is the approach followed by the 3GPP IMS Service Continuity [14] that supports terminal and session mobility. This still has the undesirable effect that applications have to be aware of the change of IP address and deal with it. This is reasonable when the terminal is mobile and it is the entity enjoying the mobility support, but it is much more undesirable for the communication peer that can be a fixed terminal or even a server of a provider (e.g. an IPTV server), and that should not be affected by the movements at the other side of the communication.

There are some other mobility solutions proposed for IMS networks, mainly terminal mobility solutions, which keep mobility procedures transparent for communication peers of mobile terminals (e.g., multimedia servers). TRIM [12] is a SIP-based mobility support solution that provides transparent terminal mobility. Mobility in TRIM is transparent both to communication peers of mobile terminals and to the applications in the mobile terminal that are kept unaware of changes in the IP address of the terminal. The downside is that TRIM requires modifications to the architecture of the terminals defined by the 3GPP. CATMISS mobility procedures are based on those defined in TRIM, but it avoids modifications to the 3GPP architecture of users' terminals (only application layer components are added). Moreover, CATMISS supports not only terminal mobility but also session mobility, and it is integrated with a context awareness system to support decision making related to mobility.

In [15] the authors propose to use an mSCTP-based proxy to support terminal mobility. The main drawback of the proposed solution is that it requires several modifications and adaptations to the IMS infrastructure. First, HSS has to be modified to handle different IP primary/secondary addresses. Second, the Register SIP message is extended with two new optional headers and a new method is added to handle handover signalling. Moreover, in the path between the mSCTP proxy in the network and the mSCTP agent in the mobile terminal, data is sent with two transport layer headers, one corresponding to the transport protocol used by the application and the other of the mSCTP protocol, which implies a waste of valuable resources in the wireless interface of the mobile terminal. The work in [16] proposes to modify Proxy-CSCFs and Interrogating-CSCFs to make them mobility-aware. The two main mobility functions incorporated in these servers are detecting the mobility of terminals and controlling an RTP proxy that makes bicasting (duplicates data flows that are sent to the old interface and the new interface) to reduce packet loss during handovers. Therefore, these previous works propose changes in the IMS framework which complicates the deployment of the solutions. The work in [17] proposes a terminal mobility solution based on combining SIP and the multihoming protocol SHIM6 operating in both ends of the communication (the mobile terminal and the multimedia server). This solution provides terminal mobility transparent to applications in IMS and it does not require modifications to the IMS framework. The problem is that it requires SHIM6 support not only in the mobile terminal, which could be a reasonable requirement to gain mobility support, but also in the communication peer of the mobile terminal. Requiring modifications in any node that may eventually communicate with a mobile terminal is an important obstacle for the deployment of this solution. Contrary to these works CATMISS does not require modifications to the IMS specifications nor to the mobile terminal architecture, it supports terminal and session mobility, and it makes both types of mobility transparent for communication peers of mobile terminals, which therefore do not require any modification either.

Regarding the use of context-aware systems in IMS networks, there are some works in the literature. In [18], the authors propose an extension to IMS to integrate a context-aware system fed by social networks information. The work in [19] extends the IMS architecture with a context management system that allows influencing the behaviour of multimedia applications, and proposes the use of a proxy to integrate generic sensors networks into this context management system. In [20] the authors propose a context-aware system integrated on top of an IPTV/IMS architecture aiming at offering personalized IPTV services. This solution relies on the core IMS architecture for transferring the different context information to a context-aware server. The work in [21] proposes the provision of personalized IPTV services based on a web 2.0 platform integrated with IMS. In [22] the authors propose a system for personalized advertising for IMS which is integrated with the IMS charging framework to reward users for viewing advertisements. Most of these mentioned works focus on content personalization (i.e., a personalized Electronic Program Guide) [20], [21], location-based services [19], or personalized advertising [22], and in the cases where context-aware mobility is considered (context state can trigger a terminal or session mobility procedure) [19] the mobility procedures to make it possible are not defined. However our paper focus on integrated mechanisms and protocols to enable personalized terminal mobility and session mobility based on context-information.

Finally, there are some proposals in the literature based on the idea of terminals getting support from the network in the process of deciding that a mobility procedure could improve the users' Quality of Experience (QoE). The work in [23] proposes to use a cross-layer solution that combines SIP with IEEE 802.21 MIH. This approach requires MIH support in IMS and the mobility context information is restricted to low layers state. For a related functionality in LTE networks, the 3GPP has proposed a new network entity called the Access Network Discovery and Selection Function (ANDSF) presented in [24] [25] and applied to network assisted flow mobility in IMS environments in [26]. The ANDSF sends to terminals information about available access networks and policies on how to choose the access network. The terminal, considering user preferences available locally, processes and applies the policies. The context-awareness system defined in CATMISS extends 
this concept. It gathers more types of information, such as user preferences, available terminals, and multimedia sessions' parameters. It also defines a generic system of context detectors to collect the context information. Having the information in the network allows applying algorithms to process it and infer new information that can help in serving the user, for example learning implicit preferences. The context information in our system allows supporting additional functionality such as session mobility.

\section{CONTEXT-AWARE MOBILITY}

In this section we present CATMISS, our solution to support context-aware mobility in IMS transparently to the communication peers of mobile terminals. It supports both terminal and session mobility. For the case of session mobility, in which a multimedia session is moved from an original device to a target device, two modes are possible: push mode, in which the mobility SIP signalling is initiated in the original device, and pull mode in which the mobility SIP signalling starts in the target device. We present the architecture of CATMISS first, followed by the description of a mobility scenario that allows us to explain the mobility procedures.

\subsection{Architecture}

The architecture of CATMISS for context-aware mobility for IMS services is presented in Fig. 1. There are three main components in the architecture: 1) The Mobility Context-Awareness System, which deals with context information; and 2) the Service Continuity Agent and 3) the UE (User Equipment) Service Continuity that handles the SIP-based mobility support. Next subsections explain the functionality of the different modules of the architecture.

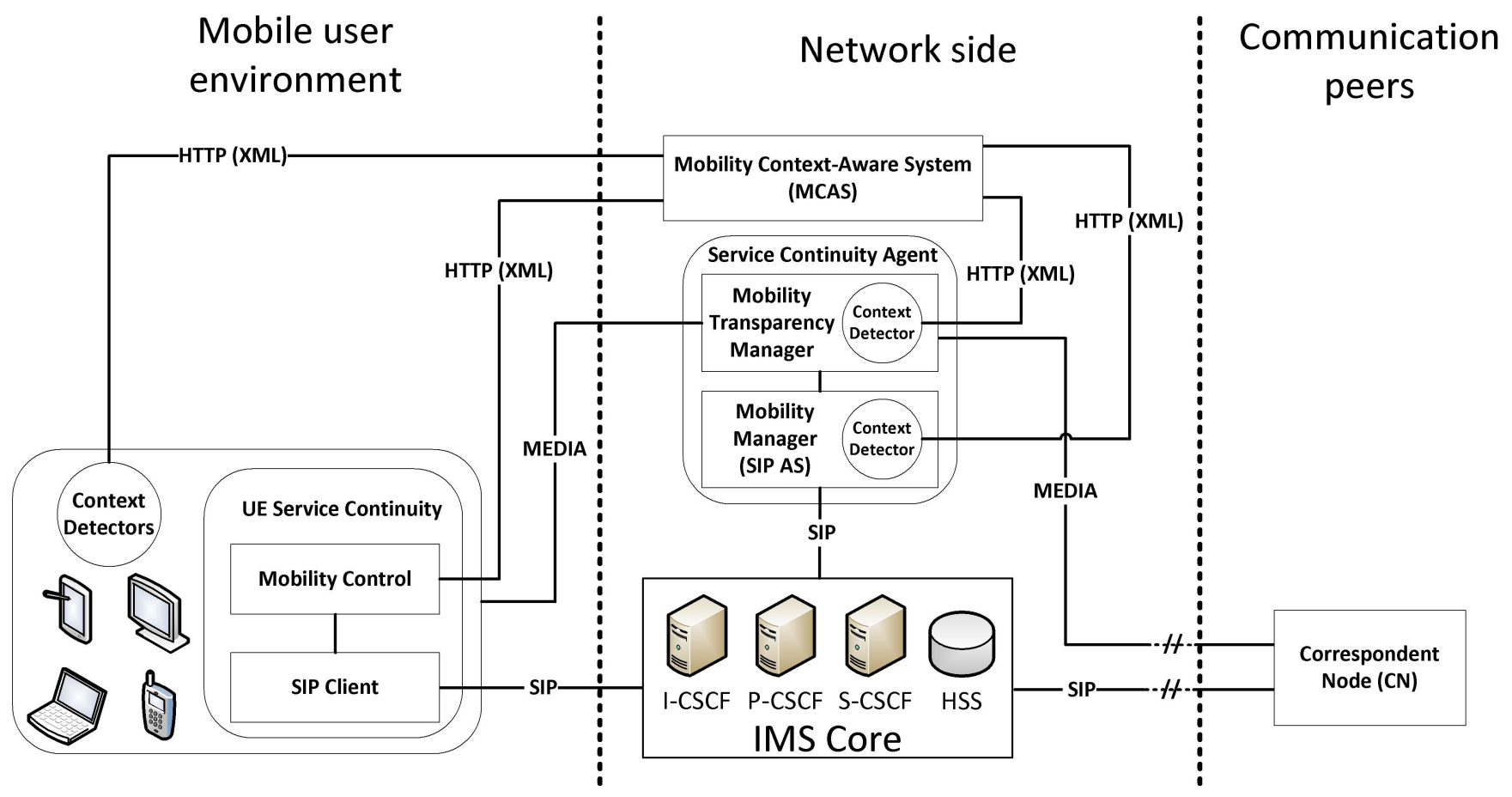

Fig. 1 CATMISS architecture

\subsubsection{Mobility Context-Awareness System \& Context Detectors}

CATMISS relies on a Mobility Context-Awareness System (MCAS) to provide users' terminals with information needed to carry out mobility procedures. The MCAS is located in the home operator network and stores context information of the user environment, devices, network and ongoing multimedia sessions that is useful to manage the different mobility procedures.

The information stored in the MCAS allows operators to enhance their users' Quality of Experience (QoE). The MCAS has three main functions: collecting context information related with mobility procedures, storing and managing that context information, and using that information to trigger mobility actions that would improve user's QoE.

The information managed in the MCAS (context information) and used to help UEs on mobility procedures is described next: 
- Possible target UEs of a session: upon moving a session from an UE to another, we need to discover potential UEs where the session can be transferred. These potential target devices are those where 1) the user is registered in the IMS, 2) the multimedia session codecs are supported and 3) any of the networks the target UE is attached to has enough resources for the ongoing multimedia session. The MCAS evaluates these conditions based on context information so that it can choose the best UE for the multimedia session.

- Active multimedia sessions of the user: the information about ongoing multimedia sessions includes 1) SIP session identifier formed by the Call-ID, "from" tag and "to" tag of the SIP dialog. This information is used to identify the SIP session to be transferred to a target UE. 2) Device identifier where the user is currently enjoying the multimedia session. 3) Addressing information of the participants in the multimedia session. 4) SDP description of the media session that includes the used codecs and the bandwidth consumed by the multimedia session.

UEs can ask the MCAS for information about possible multimedia sessions that can be transferred to a specific UE. The MCAS announces those multimedia sessions that fulfil the following conditions: 1) the potential target UE supports the codecs used in the multimedia session (MCAS stores UEs features) and 2) there is at least one access network to which the UE is attached to and that has enough resources to deal with the multimedia session (MCAS monitors network status).

- Available access networks of the UE and network status: upon terminal mobility or when moving a session from an UE to another, an access network must be chosen for the multimedia session. If the UE, or the target UE in the case of session mobility, has multiple interfaces e.g., cellular $(3 \mathrm{G} / 4 \mathrm{G})$ and Wi-Fi access interfaces, knowledge of the active network interfaces is needed in order to select which interface and access network are good candidates to be used for the multimedia session. The MCAS stores information about available access networks, with their respective technologies, in the location of the terminal, the network status in each of the access networks, and the bandwidth consumed by the multimedia session. Therefore, the MCAS can select, or help to make the selection, of a network interface and access network with enough resources to deal with the multimedia session.

The MCAS also stores users' mobility preferences, so a user configures her preferences only once and they can be consulted independently of the UE in use at a given moment. This is a desired feature in an environment in which users are expected to have several terminals and the possibility of session mobility. The user will configure mobility preferences regarding:

- Mobility initiation mode: users can select to use manual, semi-automatic or automatic mobility initiation modes. In manual mode the user decides that he wants to initiate a mobility procedure and the MCAS provides the information to the UE to support the mobility procedure. In semi-automatic mode the network (i.e., the MCAS considering the different context information related with the user, the terminals and the networks) proposes initiating a mobility procedure, but the user always has the opportunity to accept or reject this suggestion. In automatic mode the network (i.e., the MCAS taking into consideration all the context information) triggers a mobility procedure in the user terminal. When the user configures the use of the semi-automatic or automatic mobility initiation modes, UEs subscribe to mobility notifications in the MCAS. This way, the MCAS monitors context information and informs UEs when a mobility procedure is appropriate.

- User preferred devices: if the user configures semi-automatic or automatic mobility initiation mode, the selection of the preferred UE to enjoy a multimedia session is done on behalf of the user. This decision is based on a list of UEs in order of preference configured by the user, the features of the UE (e.g. screen size, codecs supported), the network status, and the parameters of the multimedia session (e.g. codecs of the multimedia session).

- Preferred access network types: if a target UE has more than one active network interface or a new access network interface becomes active on an UE, the access network that is going to be used for the multimedia session has to be selected. The user can configure in order of preference the different access network types. For example, a user could prefer to use Wi-Fi access networks instead of cellular whenever it is possible. On the other hand, the MCAS can also consider the network status when selecting an access network checking if the network fits the multimedia session needs.

In manual mobility initiation mode, the MCAS provides the information needed to support mobility procedures. For example, a user can request information about nearby UEs to select one to initiate a session mobility procedure. In automatic or semiautomatic mobility initiation modes, the MCAS uses the context information and the user preferences to proactively notify UEs when there are opportunities for terminal or session mobility that would improve user's QoE. This way, UEs (associated to users) subscribe to mobility notifications in the MCAS and the MCAS informs subscribed UEs about specific mobility procedures that would be appropriate based on the current environment conditions. Mobility triggers to which UEs can subscribe are: 
- Push session mobility trigger: this trigger informs an UE with an ongoing multimedia session about the possibility of pushing the session to another device (semi-automatic mobility) or makes the UE to initiate a push procedure to transfer the session to a target device (automatic mobility). The trigger is sent by the MCAS when it detects that the user is close to another device or devices where the user would prefer to enjoy the multimedia session. Inside the trigger message, the MCAS sends the addressing information of the target UE. This way, the receiver UE can transfer the session to the new UE. Thanks to its intelligence and the collected context information, the MCAS can check in advance that the target UE fulfil the next conditions: 1) the UE can support the multimedia session (codecs and screen size), 2) the UE is attached to an access network with enough resources for the ongoing multimedia session and 3) the user is registered in the device. If there are several devices nearby, the target UE is selected taking into account user preferences. Besides, if the target UE has several available access network interfaces, the access network interface is selected based on user preferences and network status information.

- Pull session mobility trigger: this trigger informs an UE about the possibility of pulling a multimedia session present on another device (semi-automatic mobility) or makes the UE to initiate a pull procedure to transfer the session from another device (automatic mobility). The MCAS sends this trigger to an UE when 1) the user is close to the device and registered in it, 2) the device is more preferred by the user than the current one, 3) the UE supports the multimedia session and 4) any of the networks interfaces of the UE has an access network available with enough resources for the multimedia session. The trigger message includes information about the session to be transferred and the selected access network to be used. This way, the target UE has all the information needed for pulling the multimedia session.

- Terminal mobility trigger: this trigger is sent by the MCAS when it recommends (semi-automatic mobility) or requires (automatic mobility) an UE to perform a handover to another access network because, for example, network congestion has been detected. The trigger includes information about the target access network. The MCAS selects the target access network considering network status, user preferences and parameters of the multimedia session. The MCAS keeps context information that it uses to check that the target access network has enough resources to support the multimedia session.

- Registration trigger: this trigger is useful when the MCAS detects a possible target UE for a session transfer, but the session cannot be transferred because the user is not registered in that UE yet. When the UE receives the registration trigger, the UE performs a user registration (registration data is preconfigured by the user in the terminal), so that a multimedia session can be transferred to the target terminal.

Note that in CATMISS, the MCAS gathers information, mostly from network elements (see below), and processes it to trigger the UEs just with selected information useful for their mobility procedures, i.e., it filters information. An alternative model would be to use the MCAS to recollect information and provide all of it to the UEs, so they could assess it. However, this would increase UEs complexity, which is inappropriate when UEs are limited devices (mobile devices). This way, the intelligence of the MCAS relieves UEs of this workload. Moreover, we envision the use, in the MCAS, of much more sophisticated algorithms to process the context information to learn and infer usage patterns or to allow the detection of specific situations where terminal or session mobility would improve users' QoE. An example is using learning algorithms in the MCAS to deduce the preferred device for a multimedia session with specific parameters (session type, session participants, codecs, consumed bandwidth, etc.) taking into account the devices selected by the user for his multimedia sessions in the past. Then, the MCAS can propose to transfer a session to the preferred device, adapting the circumstances to user's liking. A more complex example would be to use algorithms in the MCAS to infer associations between the devices or access network types used for multimedia sessions and different parameters such as the type of multimedia sessions, the time of the day, the participants of the session, etc. The MCAS can extend user preferences with rules inferred from the context data like "the user prefers the device X (living-room TV) for the IPTV service at night" or "the user prefers chatting in the terminal $\mathrm{Y}$ (mobile phone) in the morning using the Wi-Fi access network, but he prefers the device $\mathrm{Z}$ (tablet) in the afternoon". For instance, the MCAS detects that the user usually talks with a specific user (e.g., sip:mother@example.com) through videoconference using the device X (living-room TV) from 19:00 h. to 20:00 h. in the afternoon. This way, if the user establishes a multimedia session with similar features using another device (smartphone), the MCAS can propose to transfer the session to the living-room TV.

The key benefit of CATMISS is that this processing, that may also depend on operators' requirements, is kept in a server and does not overload the resource-limited users' devices. Additionally, the MCAS makes the context aware mobility solution independent of the terminal features. UEs do not need to store context information to decide about mobility events, the MCAS does these tasks on behalf of them, and therefore the user does not depend on using a particular terminal to achieve the benefits of our proposed context awareness system. Of course, simple terminal mobility, changing the point of attachment due to signal/coverage reasons, still can be initiated in the UE, but the MCAS enables richer mobility scenarios. 
The MCAS provides context information and triggers UEs using XML messages transported by means of the HTTP protocol. Fig. 2 shows a fragment of the XML message that informs about active multimedia sessions and network information of a user's terminal.

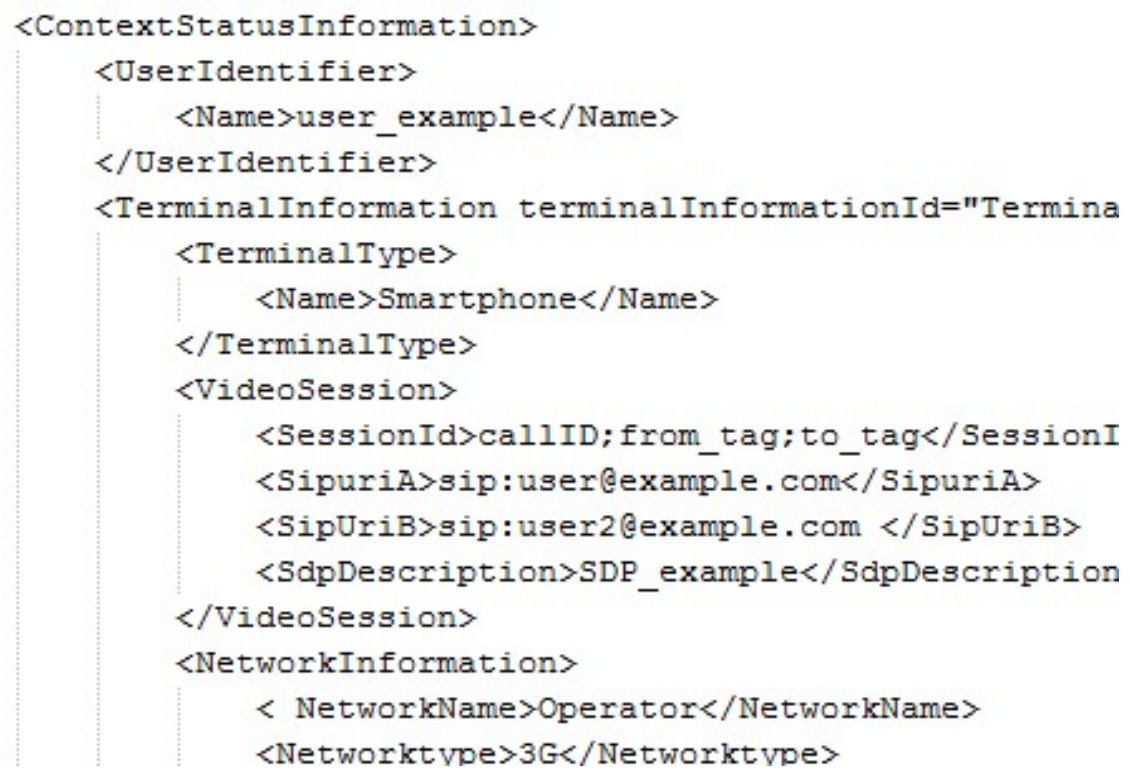

Fig. 2 XML message example with context information in CATMISS

The last function of the MCAS is to gather the mobility context information that shows the current situation of users, devices, networks and multimedia sessions. In CATMISS this context information is acquired by means of a set of different context detectors. A context detector is basically a software program that monitors some parameters and sends specific data to the MCAS. Examples of context detectors are a program in an RFID reader used to detect the presence of a user in front of a terminal that updates the MCAS whenever the user's RFID tag is read, or software in an IPTV server analysing RTCP reports of multimedia sessions to monitor packet losses. Context detectors send to the MCAS context information codified in XML messages transported by HTTP.

In CATMISS the MCAS is fed by a set of context detectors to gather the following context information:

- User identity and location. Several mechanisms can be used to detect user location. In the UP-TO-US project, three different mechanisms were used to detect the proximity of a user to terminals: a user location mechanism based on RFIDs, an alternative solution that used Near Field Communication (NFC), and an algorithm that detects the presence of the user's smartphone by means of triangulation, measuring the Wi-Fi signal strength. Other mechanisms could be easily integrated in the architecture.

- Terminals and their features. There are several ways of gathering this information but our architecture defines three mechanisms to cope with this aspect: static manual configuration, context detectors in the terminals to inform the MCAS about its characteristics, or context detectors associated with device discovery servers in access networks to inform about the characteristics of the discovered terminals.

- Network status. Collected from context detectors in network points of attachment.

- Multimedia session parameters. Collected from a context detector in a SIP Application Server (the Mobility Manager, described below) in the IMS architecture. The information is updated each time a multimedia session is established or modified.

- Multimedia session quality. Collected from a context detector in a media forwarder in the home network (the Mobility Transparency Manager, described below).

We can also collect other types of context information with the corresponding detectors. The idea is that the MCAS receives information from multiple context detectors and stores it following a data model. The context information can be adapted to operator needs and the type of service that it intends to provide to their users. For instance, an extended Context-Aware 
System (CAS) was developed within the UP-TO-US project tailored for IPTV services. The UP-TO-US CAS not only included mobility context information (what we have called MCAS) but also IPTV context information related to content information and users' consumption habits to provide a personalized IPTV service that considers the user context.

Security is important for the MCAS operation:

- Context detectors in network nodes of the operator have a security association with the MCAS to exchange information with it.

- A context detector in a user device creates a security association with the MCAS using cryptographic information related with the user subscription, and the information of the context detector is only used in the MCAS in relation with the corresponding user. The procedure is the following: the context detector contacts the MCAS to request a communication on behalf of a user; the MCAS contacts an AAA server or a Home Subscriber Server to obtain a challenge and the expected reply to the challenge for that user; and finally the MCAS sends the challenge to the context detector, if the context detector can present the right reply, then the MCAS creates a security association with it.

- If the context detector is in a network node of another operator, the context detector should feed the MCAS in its own network, and the MCAS in both networks should communicate using a security association related to the roaming agreements between operators. Further definition of this operation is left as future work.

\subsubsection{Service Continuity Agent}

In CATMISS the Service Continuity Agent is an entity located in the home network. It is inserted both in the signalling path and in the data plane of users' multimedia sessions to provide functionalities for supporting transparent mobility. The Service Continuity Agent is divided in two sub-modules: the Mobility Manager and the Mobility Transparency Manager.

The Mobility Manager (MM) is a SIP Application Server (AS) [2] inserted in the SIP signalling path of users' multimedia sessions. Users subscribed to the CATMISS service are assigned a service profile whose initial Filter Criteria (iFC) has been configured to forward SIP session establishment messages to the MM. The MM is intended to make mobility transparent to the control plane of the communication peer of the UE, that from now onwards we call Correspondent Node (CN). The MM breaks the signalling session in two parts: between the UE and the MM, and between the MM and the CN. The second signalling leg does not change, even after movements of the UE, only the first signalling leg has to be updated. So no signalling is sent to update the $\mathrm{CN}$ after movements, and the control plane of the $\mathrm{CN}$ is kept unaware of the mobility of the UE. To provide transparency also to the data plane, the MM modifies SIP messages and Session Description Protocol (SDP) payloads to make media packets of multimedia sessions travel through the Mobility Transparency Manager. Additionally, the MM controls the Mobility Transparency Manager according to the information it extracts from SIP messages. This information concerns the addressing parameters of the session participants and the session parameters included in the SDP payload. The behaviour of the Mobility Transparency Manager to provide transparency to the data plane is explained below.

CATMISS includes in the MM a context detector of the parameters of established multimedia sessions. This is an appropriate place for the context detector because the MM processes all SIP signalling messages of users' multimedia sessions. An alternative would be to place this context detector in the UE but our solution relieves UEs from the complexity of having a context detector for multimedia sessions parameters and saves the bandwidth that would be consumed in the access network by the updates of the context information sent to the MCAS each time a multimedia session changes. This is particularly useful when the UE is connected to a wireless access network with limited bandwidth.

The Mobility Transparency Manager (MTM) is a media forwarder whose functionality is to make mobility transparent to the data plane of the CN. This way, the CN is unaware also in the data plane of UE movements between different access networks (terminal mobility) or movements of sessions between devices (session mobility). The MTM is controlled by the MM according to the information extracted from SIP messages of users' multimedia sessions. When the UE moves the MTM is configured to forward each media flow received from the $\mathrm{CN}$ to the current location (access network) of the UE in case of terminal mobility or to the new UE in case of session mobility. In the opposite direction, user data received from the UE is forwarded to the $\mathrm{CN}$. This way, data packets are forwarded to the appropriate destination IP address and port according to the information provided by the MM that gets it from the SIP signalling messages. The data leg between the MTM and the CN does not change even after mobility of the UE, only the leg between the MTM and the UE has to be updated by configuring the MTM with the IP address currently used by the UE.

The result is that the $\mathrm{CN}$ always perceives and maintains the same remote addressing information regardless of handovers of the UE between different access networks (terminal mobility) or the transference of sessions between devices (session mobility). Fig. 3 describes how the MTM forwards data packets in the user plane to the appropriate destination IP address and port. 
A context detector in the MTM inspects data traffic and informs the MCAS of any event in the quality of the communication. A simple mechanism to implement this functionality is analysing RTCP reports that go through the MTM.

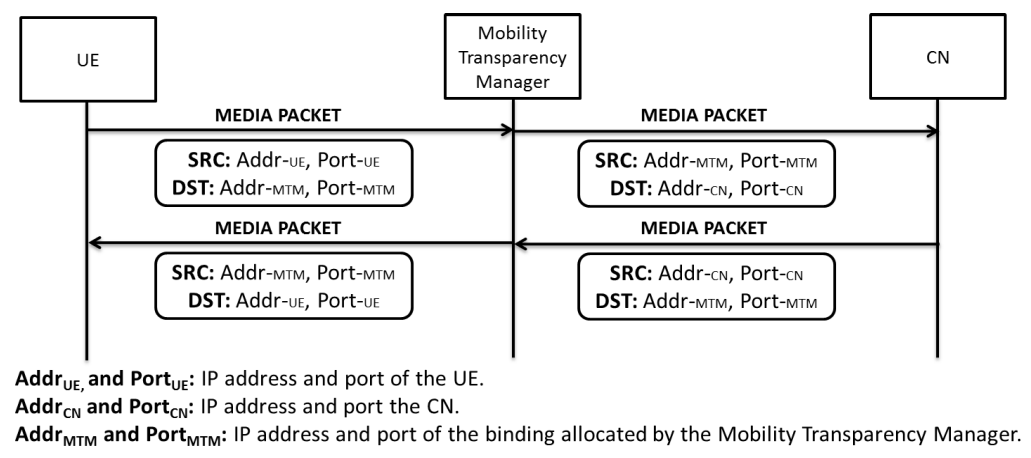

Fig. 3 Mobility Transparency Manager packet forwarding

\subsubsection{UE Service Continuity}

The UE Service Continuity module is an application layer component that CATMISS introduces in the User Equipment. The UE Service Continuity interacts with the MCAS and with the Service Continuity Agent to manage mobility procedures. The UE Service Continuity module is divided in two sub-modules: the Mobility Control and the SIP Client.

The Mobility Control sub-module has all the logic of mobility and makes mobility decisions taking into account triggers received from the MCAS. The Mobility Control sub-module subscribes to the mobility events in the MCAS which monitors user context and notifies it when a mobility event occurs. When the Mobility Control receives a trigger from the MCAS or decides to execute a mobility procedure, it requests the SIP Client to exchange with the Service Continuity Agent the appropriate SIP signalling to manage the mobility procedure. This way, the Service Continuity Agent is maintained updated with the current addressing information of the UE when it moves between access networks (terminal mobility) or the session is transferred between devices (session mobility).

The SIP Client is in charge of the SIP signalling exchange for registration, session establishment, session modification, and session release. SIP signalling messages are routed through the IMS infrastructure before arriving to the Service Continuity Agent and the CN.

\subsection{Mobility procedures}

The different context-aware mobility procedures of CATMISS are presented in this section. To explain the mobility procedures we are going to use an example. A user establishes an IPTV session to watch a live sports event when she finishes her duties at work, and while she waits for the time to go to the bus stop to get back home. We will detail how CATMISS manages the mobility while the user travels to her home. The figures in this section present the UEs used by the user in her way from the office to home (PC at work, smartphone and living-room TV at home) and the main messages exchanged among modules. This way, the CN is an IPTV platform. The MM sub-module is the Mobility Manager, and the MTM sub-module is the Mobility Transparency Manager. The MCAS is continuously gathering all possible context information from user environment and the network. We assume that the user is already registered in the IMS in the PC at work and in the smartphone.

\subsubsection{Session Establishment}

Initially, the user is at work where she initiates an IPTV session with the IPTV platform using the PC at her office. The session establishment is presented in Fig. 4. The work PC begins the session establishment issuing an INVITE request (1) to the IMS Core that according with the initial Filter Criteria (iFC) is routed (2) towards the MM. The SDP offer of the INVITE message contains the media descriptions that are processed by the MM (3). For each media component included in the SDP offer: 
a) The MM obtains the addressing information where the work PC will receive the data traffic. This is the IP address and port included in the media component description. The MM requests the MTM to create a binding for this addressing information. As result, the MTM allocates a new pair of IP address and port, and returns it to the MM.

b) The MM modifies the media description of the SDP payload replacing the IP address and port by the binding obtained from the MTM. This way, the IPTV platform will send the data traffic to the MTM.
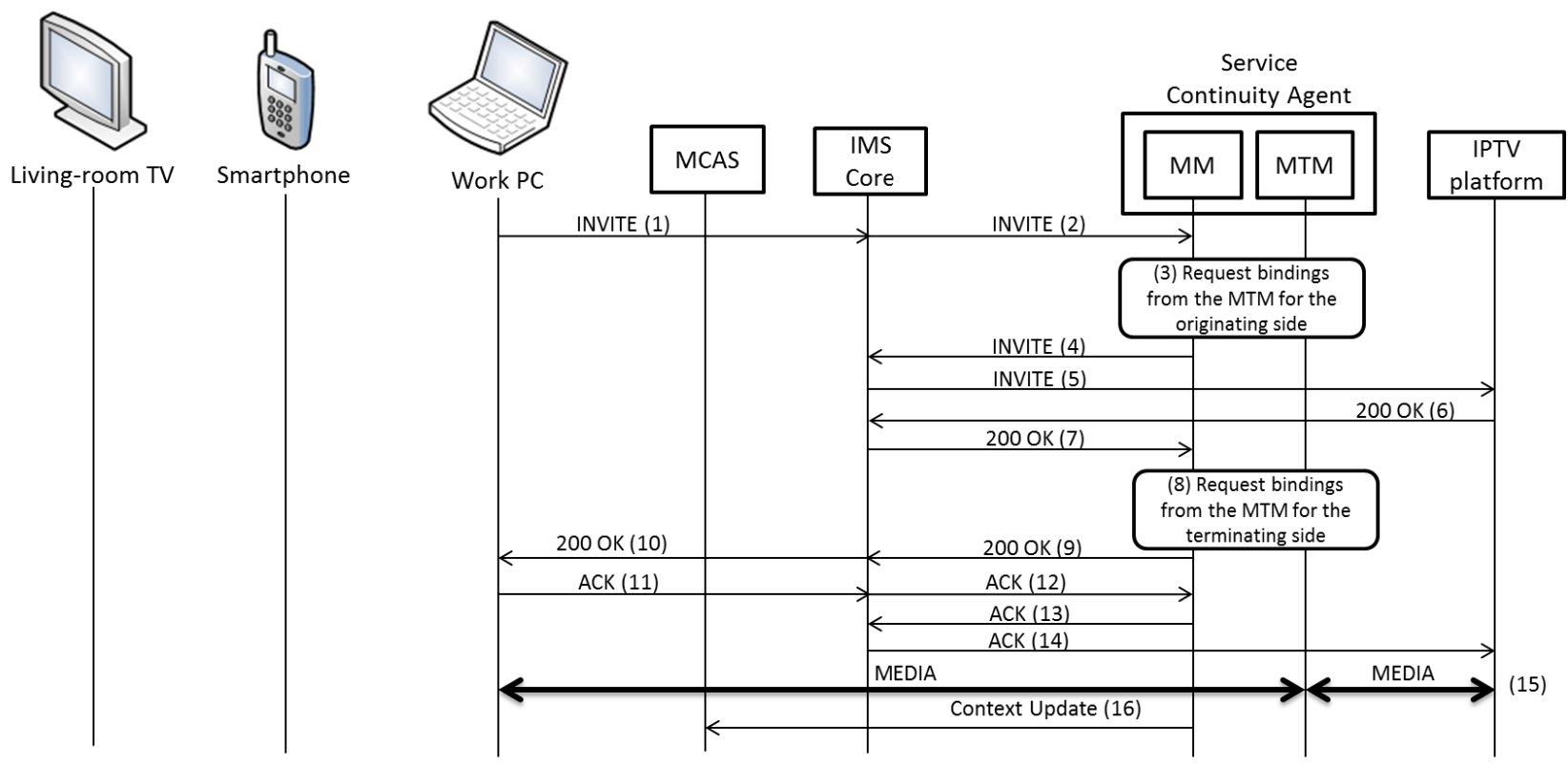

Fig. 4 Session establishment in CATMISS

After this processing, the MM issues a new INVITE message (4) that includes the modified SDP offer that is routed by the IMS core to the IPTV platform (5). Since the SDP payload received by the IPTV platform includes the IP addresses and ports of the bindings allocated by the MTM, the data traffic sent by the Media Server of the IPTV platform will reach the MTM. On the other hand, the IPTV platform generates a 200 OK response including a SDP payload with the media descriptions for the IPTV platform side that arrives to the MM (6-7). The MM processes the 200 OK message (8). For each media component included in the SDP answer:

a) The MM obtains the addressing information indicated by the IPTV platform and requests the MTM to create a binding for this addressing information. As a result, the MTM allocates a new pair of IP address and port, and returns it to the MM.

b) The MM modifies the media description replacing the IP address and port by the binding obtained from the MTM. This way, the data traffic will travel through the MTM. Note that in our example of an IPTV session, the data traffic originated in the Work PC will be RTCP packets informing of the quality and statistics of the video over RTP generated in the IPTV server.

The result is that the IPTV platform always perceives and maintains the same remote addressing information (the MTM binding) regardless the session mobility or terminal mobility of the UE at the other end of the communication. Then, the MM issues a new 200 OK message with the modified SDP payload (9-10). Finally, the work PC sends an ACK message to complete the SIP session establishment (11-14). Media flows travel through the MTM (15), which forwards data packets according to the created bindings (steps (3) and (8)).

Since the MCAS is continuously gathering all possible context information from user environment and the network, it is updated with the new IPTV session information. The MM incorporates context detector software that sends multimedia session parameters to the MCAS (16). 


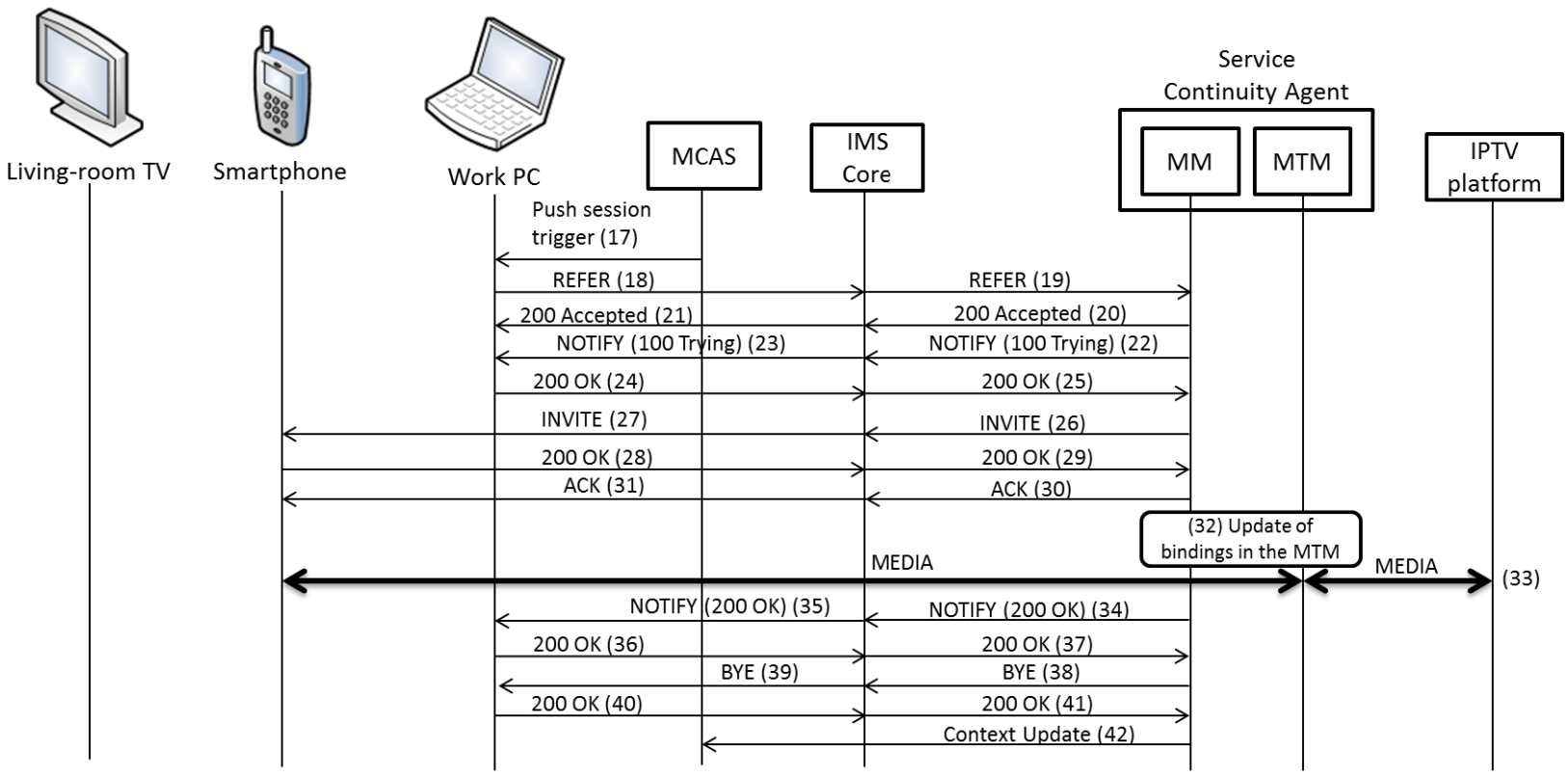

Fig. 5 Push session mobility in CATMISS

\subsubsection{Push Session Mobility}

At some point, the user decides to go home. The user trust the context awareness system and does not manually transfer the session to her smartphone, she knows that it will be done automatically on her behalf. Thanks to location detectors at work, the MCAS is aware about the user leaving her office. Then, the MCAS evaluates context information (available devices, available access networks, multimedia session parameters, etc.) and decides to transfer the session to the user's smartphone. Since Wi-Fi and 3G network interfaces are available in the smartphone, the MCAS opts for using the work Wi-Fi for the IPTV session based on user preferences and network status. Thus, a push session mobility trigger is sent to the work PC that includes all the information needed for pushing the session to the smartphone. The push session mobility procedure is presented in Fig. 5.

When the work PC receives the push session mobility trigger (17), it initiates pushing the session to the smartphone sending a REFER message [27] that arrives to the MM after being routed by the IMS Core (18-19). The REFER message includes 1) Refer-To header that indicates the destination of the session transfer (SIP URI of the user in the smartphone), 2) TargetDialog header that identifies the SIP session that has to be transferred and 3) Referred-By header that indicates who is transferring the session (SIP URI of the user in the work $\mathrm{PC}^{3}$ ). The MM processes the REFER message and informs to the work PC that it is trying to transfer the session (20-25). In order to transfer the session to the smartphone, the MM initiates a new SIP session with it. The SDP payload of the INVITE message (26) includes the bindings generated by the MTM (see step 8 in Fig. 4). After the session is established with the smartphone (26-30), the MM updates the MTM (32) with the addressing information of the smartphone extracted from the SDP payload in the 200 OK message (29). In this way, the MTM can forward the media flows to the new device (33). Note that the IPTV platform always perceives and maintains the same remote addressing information (the MTM binding) and it is not involved in the mobility SIP signalling regardless that the session is transferred between devices.

Once the session has been transferred to the smartphone, the MM informs the work PC about the success of the transference (34-37) and terminates the session with it (38-41). Finally, the context detector of the MM informs the MCAS about the transference (session has been established in the smartphone and closed in the work PC) (42).

Then the user continues watching the TV program using her smartphone connected to the Wi-Fi while she is inside the building. Note that in this paper we focus in mobility-related context information, but the system also supports triggers from the MCAS to , for example, pause or stop a video, if it detects that the user is moving and depending on user preferences. In this case, as the user is watching a life program, the preferences of the user instructs the system to continue with the reproduction of the media.

\footnotetext{
${ }^{3}$ Using Globally Routable UA URI (GRUU) [28] we can reach a user in a specific terminal.
} 


\subsubsection{Terminal Mobility}

When the user leaves the building and the smartphone is going out of Wi-Fi coverage, the MCAS, thanks to the network context detector, detects connection problems in the Wi-Fi network. This way, the MCAS sends a terminal mobility trigger to the smartphone to perform a handover to the $3 \mathrm{G}$ access network. This trigger is optional, the terminal could also detect the need for a handover due to coverage issues by itself. Fig. 6 shows the terminal mobility procedures. When the smartphone receives the terminal mobility trigger (43), it initiates the handover procedure. After obtaining IP connectivity in the new access network, it registers in the IMS to be reachable in the new IP address (44). In order to inform the MM about the new addressing information in the new access network, the smartphone sends an INVITE message (45) that includes the SDP payload updated to the new addressing information in the $3 \mathrm{G}$ access network.

When the MM processes the INVITE request, it answers with a 200 OK response (47) that includes the bindings generated by the MTM (see step 8 in Fig. 4). When the MM receives the ACK message (50) that completes the session establishment, it updates the MTM with the new addressing information of the smartphone (51). This way, the MTM can forward the media traffic to the new location of the smartphone (new access network) (52). Note that the IPTV platform always perceives and maintains the same remote addressing information (the MTM binding) regardless the smartphone UE handoffs between different access networks. Once the handover has been completed, the context detector of the MM informs the MCAS about the terminal mobility.

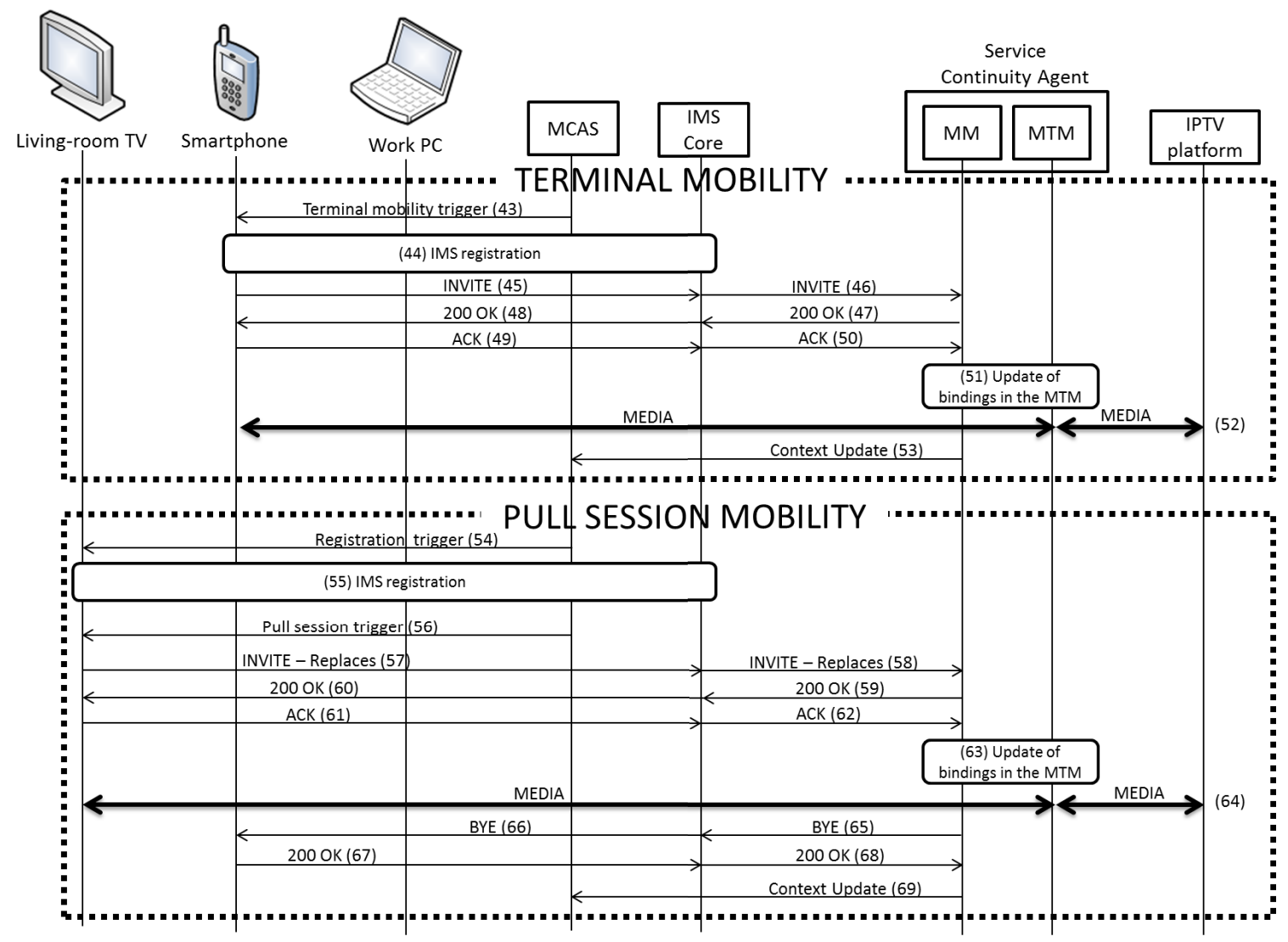

Fig. 6 Terminal mobility and pull session mobility in CATMISS

\subsubsection{Pull Session Mobility}

Then the user continues the IPTV session using her smartphone connected to the $3 \mathrm{G}$ network in her way to home. When the user gets home, her location is updated in the MCAS by the RFID system. The MCAS has learned from past actions that the user usually transfers multimedia sessions to the living-room TV when she gets home, so the MCAS decides to pull the session. The pull session mobility procedure is presented in Fig. 6. Since the user is not registered in the living-room TV, the MCAS force a user registration by sending a registration trigger (54). Once the user is registered (55), the MCAS sends a pull session mobility trigger (56) with the information needed to pull the IPTV session from the smartphone to the living-room TV. Depending on user's preferences, the trigger can only inform of the possibility of the transfer of the session (semi- 
automatic mobility). In that case, the session transfer will be initiated only when the user is ready in front of the living-room TV and accepts the trigger. Then, the living-room TV sends an INVITE request (57-58) that includes a Replaces header [29]. The Replaces header allows the MM to identify the SIP session that has to be transferred. This way, the MM sends a 200 OK response (59) including the bindings generated by the MTM (see step 8 in Fig. 4). Once the session establishment between the living-room TV and the MM has finished (57-62), the MM updates the MTM with the addressing information of the livingroom TV extracted from the SDP payload of the INVITE-Replaces message (63). This way, the MTM can forward the media packets to the living-room TV (64). Note that the IPTV platform always perceives and maintains the same remote addressing information (the MTM binding) regardless the procedure for the transfer of the session between devices. Besides, the IPTV platform is not involved in the mobility SIP signalling.

Once the session has been transferred to the living-room TV, the MM terminates the session with the smartphone (65-68) and the context detector of the MM informs the MCAS about the session mobility. Then, the user continues watching the TV program using the living-room TV.

\section{ANALYSIS OF CATMISS}

In this section we present an analysis of CATMISS. The section includes a qualitative comparison with related approaches, a study of the overhead produced by the exchange of context information, an analysis of the scalability of the solution, and a description of a prototype of an implementation of CATMISS and its evaluation.

\subsection{Qualitative Analysis}

CATMISS offers functionality and characteristics that are not present together in other approaches in the literature. In Table 1 we compare CATMISS with the related approaches that were described in sections 1 and 2 (detailed explanations are given in those sections).

Table 1- Qualitative comparison

\begin{tabular}{|c|c|c|c|c|c|c|c|}
\hline & $\begin{array}{l}\text { For IMS- } \\
\text { based } \\
\text { Networks }\end{array}$ & $\begin{array}{l}\text { Context } \\
\text {-aware }\end{array}$ & $\begin{array}{l}\text { Terminal } \\
\text { Mobility }\end{array}$ & $\begin{array}{l}\text { Session } \\
\text { Mobility }\end{array}$ & $\begin{array}{l}\text { Mobility } \\
\text { Transparent to } \\
\text { Applications } \\
\text { (in MN or CN) }\end{array}$ & $\begin{array}{l}\text { Modifications } \\
\text { to IMS } \\
\text { Infrastructure }\end{array}$ & $\begin{array}{l}\text { Modifications } \\
\text { to the 3GPP } \\
\text { Terminal } \\
\text { (MN or CN) } \\
\text { Architecture }\end{array}$ \\
\hline GTP [4], PMIP [5] & Yes & No & $\begin{array}{l}\text { Localized } \\
\text { domain }\end{array}$ & No & $\mathrm{MN} \& \mathrm{CN}$ & No & None \\
\hline MIPv4 [6], MIPv6 [7] & No & No & Yes & No & $\mathrm{MN} \& \mathrm{CN}$ & -- & $\mathrm{MN}$ \\
\hline IMS SC [14] & Yes & No & Yes & Yes & None & No & None \\
\hline TRIM [12] & Yes & No & Yes & No & $\mathrm{MN} \& \mathrm{CN}$ & No & $\mathrm{MN}$ \\
\hline $\begin{array}{l}\text { Huu et al. [15] } \\
\text { mSCTP-based Proxy }\end{array}$ & Yes & No & Yes & No & $\mathrm{MN} \& \mathrm{CN}$ & Yes & $\mathrm{MN}$ \\
\hline Arnaud et al. [16] & Yes & No & Yes & No & $\mathrm{CN}$ & Yes & None \\
\hline $\begin{array}{l}\text { Achour et al. [17] } \\
\text { SIP-SHIM-based Solution }\end{array}$ & Yes & No & Yes & No & $\mathrm{MN} \& \mathrm{CN}$ & No & $\mathrm{MN} \& \mathrm{CN}$ \\
\hline $\begin{array}{l}\text { Context-Aware IMS systems } \\
{[18][19][20][21][22]}\end{array}$ & Yes & Yes & No & No & -- & No & None \\
\hline $\begin{array}{l}\text { Choong et al. [23] } \\
\text { SIP\&802.21 MIH }\end{array}$ & Yes & Partially & Yes & No & None & No & None \\
\hline Vidal et al. [26] & Yes & Partially & $\begin{array}{l}\text { Flow } \\
\text { Mobility }\end{array}$ & No & $\mathrm{MN} \& \mathrm{CN}$ & No & $\mathrm{MN}$ \\
\hline CATMISS & $\underline{\text { Yes }}$ & $\underline{\text { Yes }}$ & $\underline{\text { Yes }}$ & $\underline{\text { Yes }}$ & $\underline{\mathrm{CN}}$ & $\underline{\text { No }}$ & None \\
\hline
\end{tabular}

As shown in Table 1 CATMISS is the only solution that combines the following characteristics: (1) it is a mobility support solution for IMS services; (2) CATMISS is context-aware and so decisions take into account context information about the 
users, the services and the network; (3) it does not only support terminal mobility but also session mobility; (4) it provides transparent mobility to the correspondent node (e.g., a fixed terminal such as an IPTV server) that is not affected by the movements of the other side of the communication; (5) CATMISS does not introduce any modifications to the IMS infrastructure and (6) it does not need modifications to the communications stack of the MN nor the $\mathrm{CN}$ (so modifications in the kernel of those types of nodes are not needed).

\subsection{Analysis of the overhead of the exchange of context information}

In order to study the feasibility of CATMISS, a first step is to analyse the overhead produced by the exchange of context information between the MCAS, the context detectors and the Mobility Control sub-module of the UE. As mentioned above, CATMISS includes a context detector in the Mobility Manager (MM) that is in charge of sending multimedia session information to the MCAS. Since both the MCAS and the MM are located in the core network of the home network operator, the overhead produced by this communication can be considered negligible due to the high bandwidth available between core network components. The relevant overhead is the one produced by the exchange of context information over the radio access link since it is the most resource-bounded link. This concerns the different triggers sent by the MCAS to the Mobility Control module (see Fig. 4, Fig. 5 and Fig. 6). We calculated that the data traffic needed for sending an XML trigger similar to the one shown in Fig. 2 inside an HTTP request is about 1.5 Kbytes (note that the size of the trigger message can vary depending on the number of sessions, terminals and access networks). The rate of triggers depends on the scenario and the particular situation but as triggers are related to mobility events, the rate is necessarily limited. We can assume that even in a highly dynamic scenario, we cannot expect rates higher than one trigger per minute for each user. The number of users in a cell depends on the technology and configuration but it is also usually related to the available bandwidth (to give service to more users we need more available bandwidth). Taking as an example a $3 \mathrm{G}$ cell, a reasonable maximum number of users is 52 [30]. Then, on average, the total bandwidth consumed by sending triggers to those users each minute is $10.6 \mathrm{Kbits} / \mathrm{s}$, which is reasonable for our example that is a worst case. Thus, taking this example as reference we can say that the overhead does not compromise the radio access link.

\subsection{Scalability of CATMISS}

Another aspect to be considered in the feasibility of the proposed architecture is how it can cope with an increase in the number of simultaneous mobile terminals using the mobility support functionality. The network elements that will have to attend the increased workload are the Mobility Context-Awareness System (MCAS), the Mobility Manager (MM), and the Mobility Transparency Manager (MTM). The key to guarantee the scalability of CATMISS is its ability to distribute the functions of each of those network elements in different physical nodes. The MM that is going to provide mobility support to a particular user is contacted by the S-CSCF assigned to the user in the IMS core. The particular S-CSCF that is assigned to a user is chosen when he registers in IMS and, in that way, the load of the S-CSCF functionality can be distributed to different physical nodes. The operator network can have several MMs and assigns a particular one to a user in his corresponding IMS user profile. The S-CSCF will contact this MM when the user establishes his first session after registering in IMS. So the load of different users can be distributed to different MMs installed in separated physical nodes.

The MTM, which takes care of the data plane of a user session, is chosen by the MM when the session is established. Several MTMs can be available in the network, and the data traffic of different sessions can be assigned to different MTMs. This is done by the MM, using any criteria of load balancing according to operator needs. So both the MTM and MM functionality can be distributed in different physical nodes, and therefore the architecture can be dimensioned to take care of an increase in the number of active users.

The distribution of the MCAS function is more challenging because some context information can be needed in relation with different users (e.g. access network information). We can use the information model of the context information to divide the information in different physical nodes, so that access to some information can require a request to a different server. Each user will be assigned a front-end MCAS, which can be different for different users, to manage the interface of that user with the context-aware system. Therefore, while navigating the context information of a user, we can have a reference to another physical node that contains information common to several users such as access network information.

\subsection{Experimental evaluation}

We have deployed a testbed with a CATMISS prototype to evaluate its functionality. The testbed allows establishing a videoconference between a user terminal and a $\mathrm{CN}$, and move the session between different devices or network interfaces (session mobility and terminal mobility) when receiving mobility triggers from the MCAS. The testbed is depicted in Fig. 7. 


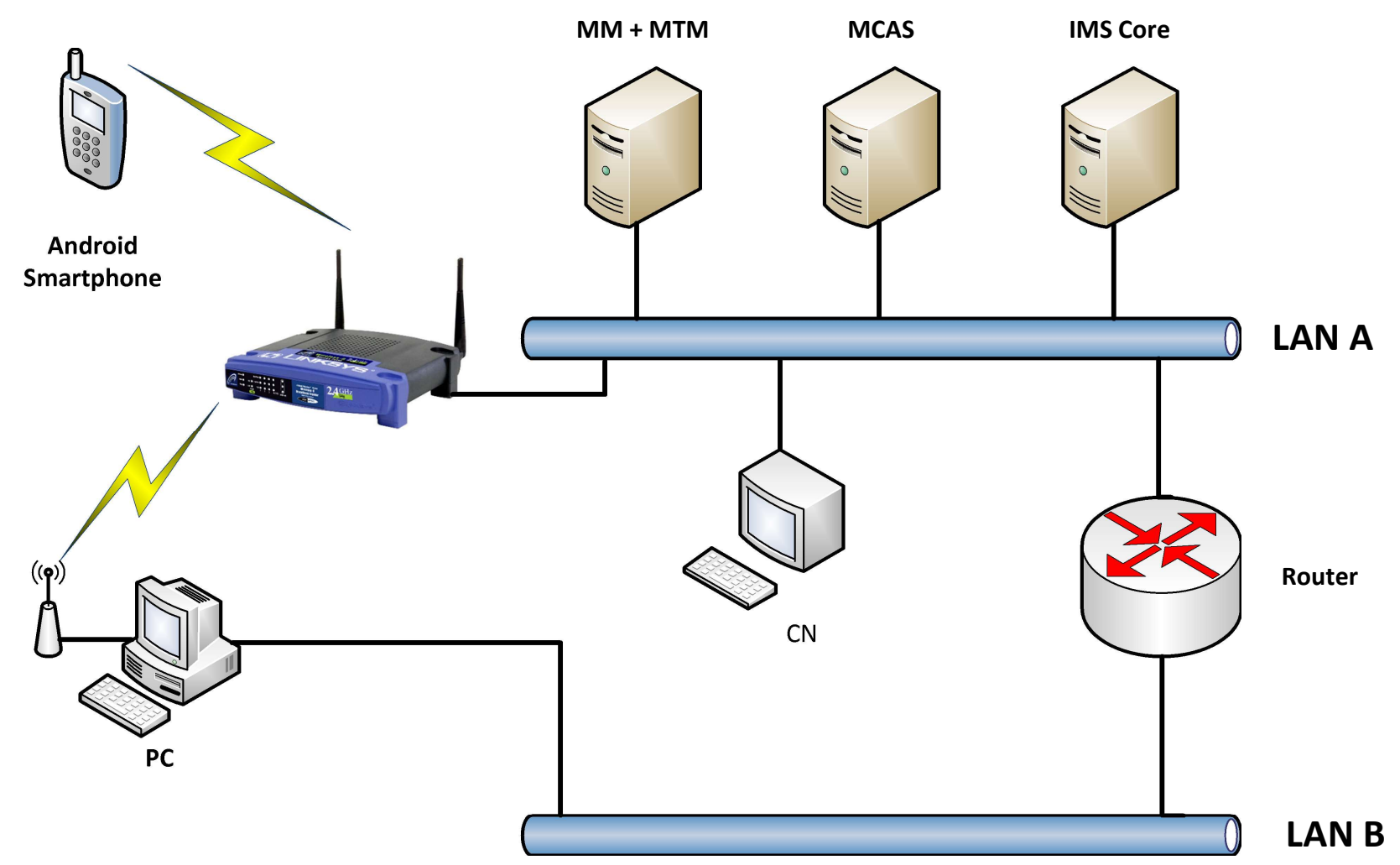

Fig. 7 CATMISS testbed

As IMS platform we use the well-known open source implementation Fokus Open IMS Core ${ }^{4}$. The IMS Core has been deployed on a Linux Ubuntu 9.04 virtual machine. Two IMS subscriptions have been created on the platform, one that represents a user in the $\mathrm{CN}$, and the other one for the user subscribed to the CATMISS service. The initial filter criteria (iFC) of the mobility service have been configured to forward SIP session establishment messages to the MM.

The MM has been developed as an application deployed in a Mobicents JAIN SLEE Server ${ }^{5}$, an open-source implementation of the JAIN SLEE 1.1 specification $^{6}$. The MTM is a stand-alone Java application that uses Java sockets to redirect media flows according to the configuration provided by the MM. The MM controls the MTM through an RMI Java interface. Both, the MM and the MTM have been deployed on a Linux Ubuntu 10.04 computer. The Mobility Context-Aware System (MCAS) is a Java application running on another Linux Ubuntu 10.04 virtual machine that interacts with a MySQL database that stores context information. The MCAS offers an HTTP REST interface to update the context information and to send mobility triggers to terminals. The $\mathrm{CN}$ is an instance of the IMS softphone myMonster ${ }^{7}$ running in a separate Linux Ubuntu 10.04 machine. Note that no modifications are required in the softphone as CATMISS keeps mobility transparent to the CN. The machines where all these entities (MM/MTM, MCAS, IMS core and CN) are deployed are connected to LAN A.

On the user side there are two different devices: a Samsung Galaxy S II smartphone and a standard PC. The Samsung Galaxy $\mathrm{S}$ II is running an IMS Client called IMSDroid IMS softphone ${ }^{8}$ that has been extended to support the SIP messages required to push a session to the PC and to pull an active session from the PC. The smartphone is connected through Wi-Fi to a Linksys WRT 54GL Wi-Fi router attached to LAN A.

\footnotetext{
${ }^{4}$ Open IMS Core, Fraunhofer Institute for Open Communication Systems (http://www.openimscore.org/)

${ }^{5} \mathrm{http}: / /$ www.mobicents.org/slee/intro.html

${ }^{6} \mathrm{http}: / / \mathrm{www} . j \mathrm{cp}$. org/en/jsr/detail?id=240

${ }^{7} \mathrm{http}: / /$ www.monster-the-client.org/

${ }^{8} \mathrm{http}: / /$ code.google.com/p/imsdroid/
} 
Regarding the PC, it is a Linux Ubuntu 10.04 machine running a Java application that receives HTTP requests (the mobility triggers) and launches SIPp ${ }^{9}$ scripts in order to exchange the appropriate SIP signalling to carry out the mobility procedures. The SIPp scripts are used both to push a session to the smartphone and to pull an existing session from the smartphone, depending on the trigger received from the MCAS. Totem ${ }^{10}$, the official player of the Gnome Desktop Environment, is used to reproduce the videoconference media in the PC. The PC has two network interfaces, an Ethernet network interface connected to LAN B and a Wi-Fi network interface connected to the Linksys WRT 54GL Wi-Fi router that gives access to LAN A. This way, it is possible to perform a terminal mobility procedure between these two network interfaces.

Using the described platform, the behaviour of CATMISS has been assessed. The MCAS sends the mobility triggers that automatically initiate the mobility procedures. If a pull or push session mobility trigger is received, the videoconference session of the user is transferred between the Samsung Galaxy S II and the PC. If a terminal mobility trigger is received in the $\mathrm{PC}$, the videoconference communication is moved between the network interfaces of the PC.

In order to describe the behaviour of the pull session mobility procedure, a video call is established between the $\mathrm{CN}$ and the android smartphone. Consequently, and according to the procedures presented in this paper, the information of the session is stored in the MCAS. After a while, a pull session mobility trigger including the necessary session information is issued from the MCAS to the PC. When the Java application running on the PC receives the pull session mobility trigger, it executes the appropriate SIPp scripts to exchange the signalling that causes the mobility of the session from the android device to the PC (to its Wi-Fi interface). Fig. 8 shows, for this session transfer experiment, the throughput of RTP packets received in the smartphone and in the PC, including audio traffic (ITU-T G711 codec) and video traffic (ITU-T H.263 codec). The data were obtained using the Wireshark ${ }^{11}$ network protocol analyser, capturing the traffic in the Wi-Fi network, and counting the number of RTP packets in each second of the session addressed to the smartphone and addressed to the PC (using the IO Graph tool in Wireshark). As it can be seen in the figure, the transfer of the session happens in the $33^{\text {rd }}$ second, in which we receive the last data packets in the smartphone and we start receiving data traffic in the PC. We have repeated the pull session mobility procedure 30 times to measure its average delay. This delay is defined as the time elapsed from the moment the mobility trigger is sent until the first data packet is delivered to the new device (the PC). The measured average pull session mobility delay was $1.053 \mathrm{~s}$. As we discuss below, most of this delay is caused by the time it takes to start sending the SIP signalling after receiving the mobility trigger.

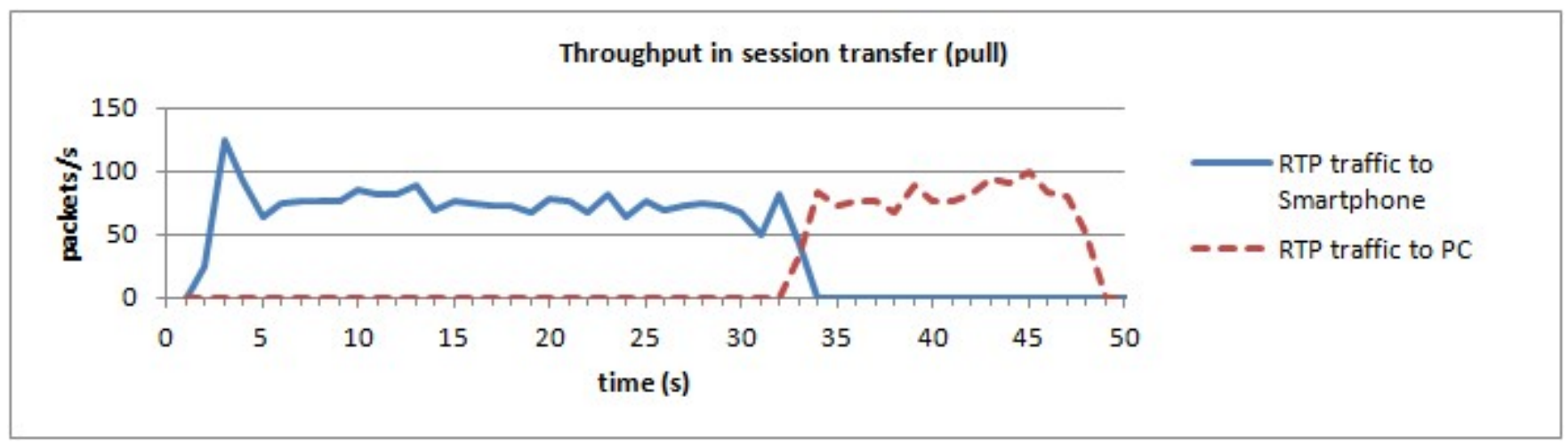

Fig. 8 Pull session mobility, throughput in the data plane

In the case of push session mobility, a video call is established between the CN and the PC (to its Wi-Fi interface). Since the user is registered in the android smartphone and the MCAS stores the context information about it, after a while, a push session mobility trigger containing the information of the android device is sent from the MCAS to the PC. That leads to the exchange of the SIP signalling that results in the session being transferred to the android smartphone.

\footnotetext{
${ }^{9} \mathrm{http}: / /$ sipp.sourceforge.net/

${ }^{10} \mathrm{https}: / /$ projects.gnome.org/totem/

${ }^{11} \mathrm{http}: / /$ www.wireshark.org/
} 


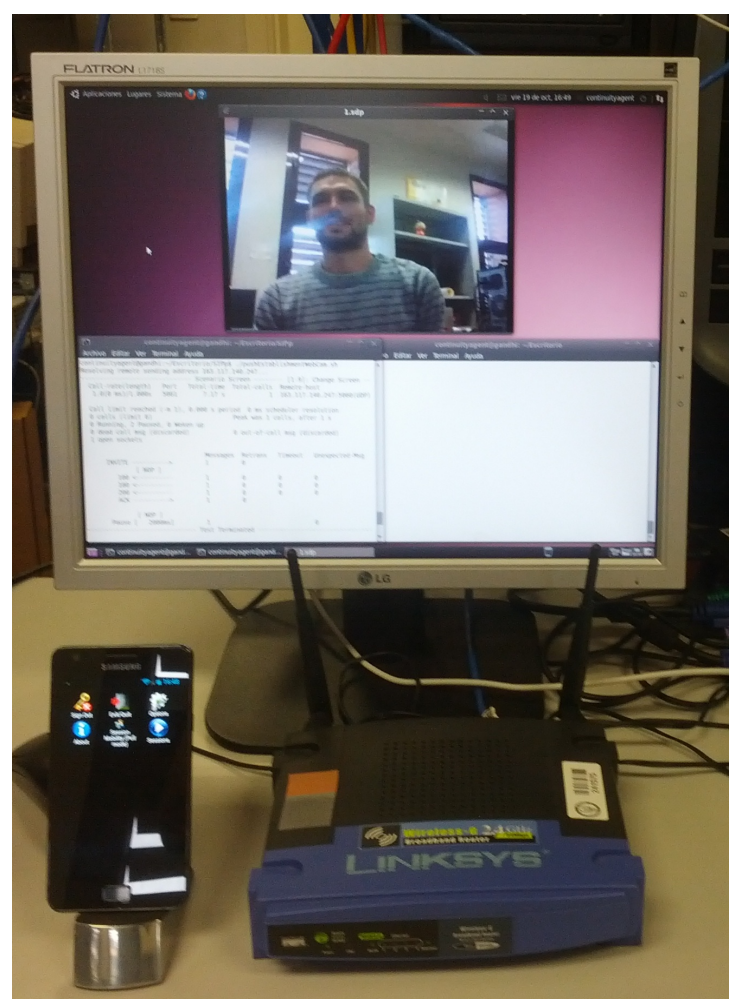

Fig. 9 Push session mobility: Initial snapshot

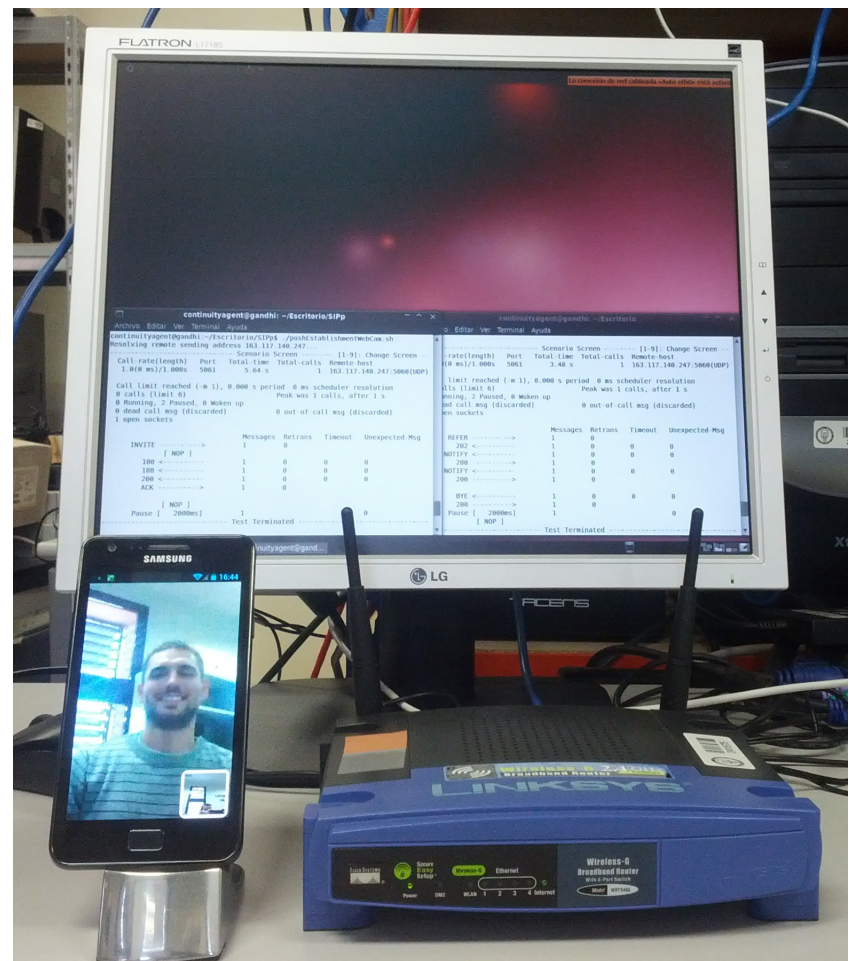

Fig. 10 Push session mobility: Final snapshot

Fig. 9 and Fig. 10 show this process. In Fig. 9, we can observe on the PC the video call established with the CN, and the android device with no active sessions. Upon the reception of a push session mobility trigger from the MCAS, the PC pushes 
the videoconference to the android smartphone. This is shown in Fig. 10, where the video call is now established on the android device and the PC shows the logs of the SIP signalling.

The delay of the push session transfer procedure is defined as the time elapsed from the moment the MCAS sends the mobility trigger until the first data packet is received in the android device (target device). Repeating this measurement 30 times we have obtained an average value of $1.358 \mathrm{~s}$ for the push session transfer delay. As before, the most significant part of this delay is the time it takes to start sending the SIP signalling after receiving the mobility trigger. On the other hand, the push session transfer delay is longer than the pull session transfer delay. This is because the push procedure is more complex and involves more signalling (see section 3.2) than the pull procedure.

Finally we analyse the terminal mobility procedure. A video call is established between the $\mathrm{CN}$ and the PC over the Wi-Fi interface. After some time the MCAS issues a terminal mobility trigger to the PC to perform a handover from the Wi-Fi to the Ethernet access network. Upon reception of the terminal mobility trigger, the PC exchanges the appropriate SIP signalling and the media of the videoconference is changed to the Ethernet interface. Capturing the traffic with Wireshark in the PC (in all the interfaces) we can show the traffic exchanged in the session and during the handover. In Fig. 11 we show the RTP traffic (video and audio) received at the PC during the session, separating the traffic received in the Wi-Fi interface from the traffic received in the Ethernet interface. The handover takes place in the $90^{\text {th }}$ second, in which we receive the last packets in the Wi$\mathrm{Fi}$ interface and start receiving data traffic in the Ethernet interface. To see this procedure in more detail we get a measurement of the data packets, SIP packets, and the TCP packets corresponding to the mobility trigger (exchanged with the MCAS), in an interval that includes the handover. This is shown in Fig. 12. Note that in this figure we show the number of packets each $0.1 \mathrm{~s}$, to increase the resolution. As it can be seen, the trigger is received in the $88.9^{\text {th }}$ second. This starts the SIP signalling to initiate the handover in the $90^{\text {th }}$ second, while we are still receiving the RTP traffic in the Wi-Fi interface. Finally, in the $90.1^{\text {st }}$ second we are already receiving the RTP traffic in the Ethernet interface. As we can see, in this experiment the time elapsed from the moment the mobility trigger is received until the moment in which the PC starts sending SIP traffic is $1.1 \mathrm{~s}$ (with a $0.1 \mathrm{~s}$ resolution). On the other hand, once the PC starts the SIP signalling, the handover delay is less than $0.1 \mathrm{~s}$ (maximum resolution in the figure). This was also observed in the session mobility procedures. We have determined that the delay in sending SIP messages is due to the SIPp implementation that introduces a delay before sending the first SIP message, probably because of the procedure of initializing the SIP stack. To check this behaviour we did a simple experiment in a standalone machine, first sending a control message (ICMP) and then starting a SIP session using SIPp, observing a similar delay between the control message and the start of the SIP signalling.

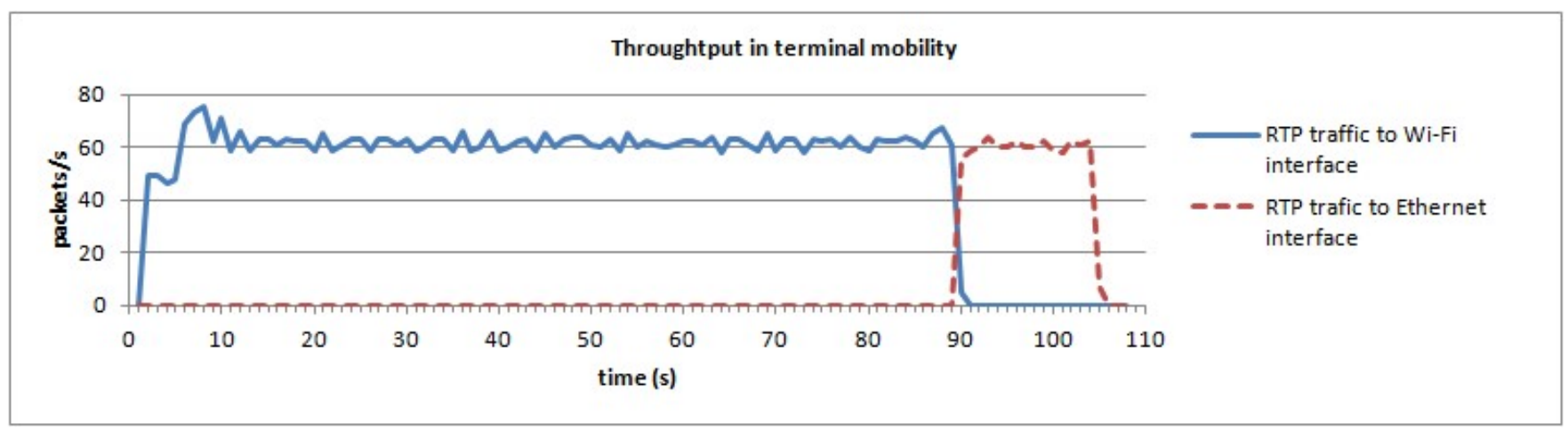

Fig. 11 Terminal mobility, throughput in the data plane 


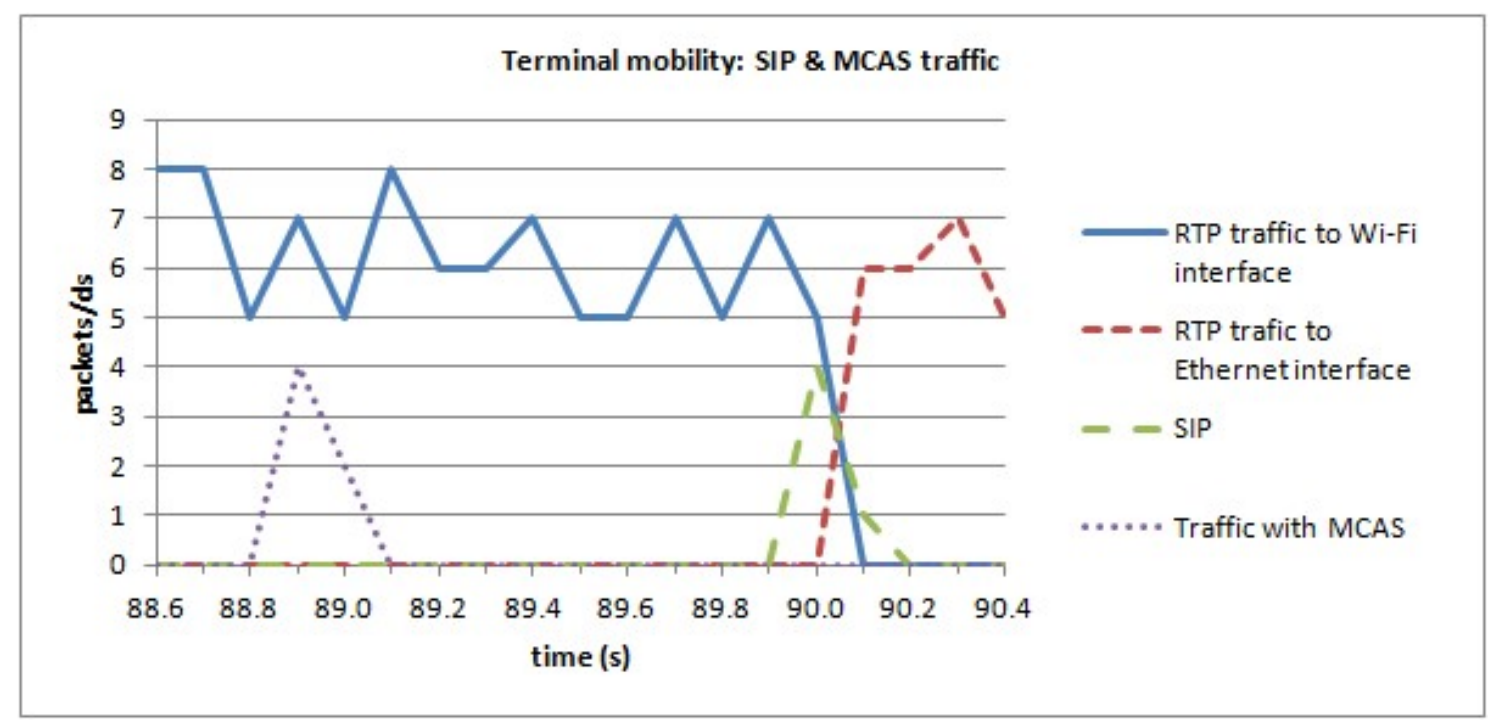

Fig. 12 SIP and MCAS traffic during handover

Then, and to get a more systematic evaluation of the performance, we have taken two complementary measurements for the terminal mobility procedure:

1) The delay of the whole terminal mobility procedure considered as the time elapsed from the moment the MCAS sends the terminal mobility trigger until the first data packet is received through the new access network interface. The average of this terminal mobility delay measured in 30 repetitions of the experiment was $1.065 \mathrm{~s}$.

2) The terminal handover delay, which is important because it could affect the quality perceived by the user, and it is the time elapsed since the UE starts the terminal mobility procedure issuing the INVITE message (after the reception of the terminal mobility trigger from the MCAS) until the first data packet is received through the new access network interface. After repeating the experiment 30 times, the average handover delay was $50.1 \mathrm{~ms}$. This second delay is, of course, part of the first one. This delay is good enough even for handovers in which the terminal uses one network interface, disconnecting from one access network and connecting to a new one, so the $50.1 \mathrm{~ms}$ becomes an interruption time. Note, however, that in practice, this value will depend on the distance between the terminal and the MM that processes the mobility SIP signalling in the network, and the workload in the SIP nodes that process the signalling. Also, in a handover with one network interface, to calculate the total handover delay we need to add the layer-2 handover delay to the handover delay caused by the SIP signalling.

As a summary of our performance results, the average delays with their $95 \%$ confidence intervals for pull session mobility, push session mobility and terminal mobility procedures are presented in Table 2.

Table 2- Delay measurement results

\begin{tabular}{|c|c|c|}
\hline Procedure & Delay (seconds) & $\begin{array}{c}\text { 95\% confidence } \\
\text { interval }\end{array}$ \\
\hline Pull session mobility & 1.053 & \pm 0.006 \\
\hline Push session mobility & 1.358 & \pm 0.054 \\
\hline Terminal mobility (whole procedure) & 1.065 & \pm 0.019 \\
\hline Terminal mobility (handover) & 0.0501 & \pm 0.0041 \\
\hline
\end{tabular}


Additionally, the prototype of CATMISS was integrated with the IPTV platform of the UP-TO-US project to provide continuity of the ongoing IPTV sessions, enhancing the user's IPTV experience ${ }^{13}$. This way, IPTV sessions can be transferred between different available end-devices including Set-Top Boxes, laptops, hand-held terminals and so on.

\section{CONCLUSION}

In this paper we have described CATMISS, a context-aware SIP-based transparent mobility support solution for IMS multimedia services that does not require any modification to the IMS specifications. Furthermore, CATMISS supports terminal mobility, and also session mobility which allows the transfer of ongoing sessions to new target terminals. The mobility context-aware system is a key component in CATMISS, providing the intelligence and information to assist users' terminals in taking mobility decisions with the aim of enhancing their mobility experience. The context-aware system stores the context information needed to support different types of mobility and it can trigger the users' terminals to recommend, or even execute, mobility procedures.

A prototype of CATMISS has been implemented using standard tools and platforms such as the Fokus Open IMS Core as IMS Platform, the Mobicents server to deploy the SIP application server providing mobility functionality, and a java application with a MySQL database to implement the context-aware system. We have carried out different tests that show that our solution works as expected, exhibiting good performance while keeping the mobility procedures transparent to communication peers of the mobile terminals, which can be standard multimedia servers (e.g., IPTV media servers).

As future work we plan to extend our architecture, including the security issues, for the case where several operators, with the corresponding roaming agreements, collaborate to enhance users QoE, which implies that mobility procedures may involve elements of different operators.

\section{ACKNOWLEDGMENTS}

The work described in this paper has received funding from the Spanish Government through project I-MOVING (TEC2010-18907) and through the UP-TO-US Celtic project (CP07-015).

\section{REFERENCES}

[1] 3GPP (June 2013) IP Multimedia Subsystem (IMS); Stage 2, TS 23.228, v12.1.0, Release 12. 3rd Generation Partnership Project (3GPP)

[2] Rosenberg J, Schulzrinne H, Camarillo G, Johnston A, Peterson J, Sparks R, Handley M, Schooler E (Jun. 2002) SIP: Session Initiation Protocol, RFC 3261. Internet Engineering Task Force

[3] 3GPP (June 2013) IP multimedia call control protocol based on Session Initiation Protocol (SIP) and Session Description Protocol (SDP); Stage 3, TS 24.229, v12.1.0, Release 12. 3rd Generation Partnership Project (3GPP)

[4] 3GPP (June 2012) General Packet Radio Service (GPRS); Service description; Stage 2, TS 23.060, v11.2.0, Release 11. 3rd Generation Partnership Project (3GPP)

[5] Soto I, Bernardos CJ, Calderon M, Melia T (September 2010) PMIPv6: A Network-Based Localized Mobility Management Solution. Internet Protoc J 13 (3):2-15

[6] Perkins C (Nov. 2010) IP Mobility Support for IPv4, Revised, RFC 5944. Internet Engineering Task Force

[7] Perkins C, Johnson D, Arkko J (Jul. 2011) Mobility Support in IPv6, RFC 6275. Internet Engineering Task Force

[8] Chiba T, Yokota H, Dutta A, Chee D, Schulzrinne H (2008) Performance analysis of next generation mobility protocols for IMS/MMD networks. In Wireless Communications and Mobile Computing Conference, IWCMC'08, pp 68-73

[9] Renier T, Larsen K, Castro G, Schwefel H (2007) Mid-session macro-mobility in IMS-based networks. IEEE Veh Technol Mag 2 (1):20-27

[10] Vidal I, Garcia-Reinoso J, de la Oliva A, Bikfalvi A, Soto I (2010) Supporting mobility in an IMS-based P2P IPTV service: A proactive context transfer mechanism. Comput Commun, 33 (14):1736-1751

\footnotetext{
${ }^{13}$ A video of a demonstration of the UP-TO-US project showing the application of CATMISS to provide contextualized IPTV Service Continuity is available at http://www.youtube.com/watch?v=eCkhfNQUeeI
} 
[11] Shacham R, Schulzrinne H, Thakolsri S, Kellerer W (Oct. 2009) Session Initiation Protocol (SIP) Session Mobility, RFC 5631. Internet Engineering Task Force

[12] Vidal I, de la Oliva A, Garcia-Reinoso J, Soto I (May 2011) TRIM: An architecture for transparent IMS-based mobility. Comput Networks 55 (7):1474-1486

[13] Sandonis V, Fernandez I, Soto I (2012) SIP-Based Context-Aware Mobility for IPTV IMS Services. In UP-TO-US Workshop, EuroITV Conference 2012 Adjunct Proceedings, pp 271-280

[14] 3GPP (Jun. 2011) IP Multimedia Subsystem (IMS) service continuity; Stage 2, TS 23.237 v11.1.0 Release 11. 3rd Generation Partnership Project (3GPP)

[15] Nguyen Huu T, Nguyen Tai H, Tran Ngoc L, Tran Quang T, Do H, Magedanz T (June 2008) mSCTP-based proxy in support of multimedia session continuity and QoS for IMS-based networks. In Second International Conference on Communications and Electronics, ICCE 2008, pp 162-168

[16] Arnaud J, Negru D (July 2012) Mobility-aware P/I-CSCF: A solution for achieving seamless handover in IMS. In IEEE Symposium on Computers and Communications, ISCC 2012, pp 43-48

[17] Achour A, Haddadou K, Kervella B, Pujolle G (July 2012) A SIP-SHIM6-based solution providing interdomain service continuity in IMS-based networks. IEEE Commun Mag 50 (7):109-119

[18] Hasswa A, Hassanein H (2011) SocioSpace: An adaptive service-oriented architecture that integrates smart spaces and social networks through the IP Multimedia Subsystem. In IEEE Symposium on Computers and Communications, ISCC 2011, pp 85-90

[19] Doolin K, Pashalidis A, Kassler A, Mota T (Feb 2008) Context-aware multimedia services in a pervasive environment: the Daidalos approach. In Proceedings of the 2008 Ambi-Sys workshop on Software Organisation and MonIToring of Ambient Systems, SOMITAS '08

[20] Song S, Moustafa H, Afifi H (2010) Personalized TV service through employing context-awareness in IPTV/IMS architecture. In Proceedings of the Third international conference on Future Multimedia Networking, FMN'10, pp 75-86

[21] Choi JK, Myoung Lee G, Park H-J (Aug. 2008) Web-Based Personalized IPTV Services over NGN. In Proceedings of 17th International Conference on Computer Communications and Networks, ICCCN '08, pp 1-6

[22] Wilson PR, Oziyani VG, Ventura N (Oct. 2009) Monetizing IMS-based IPTV through personalized advertising. In International Conference on Ultra Modern Telecommunications \& Workshops, ICUMT '09, pp 1-7

[23] Choong KN, Kesavan VS, Ng SL, Carvalho F, Low ALY, Maciocco C (2007) SIP-based IEEE802.21 media independent handover - a BT Intel collaboration. BT Technol J 25 (2):219-230

[24] 3GPP (March 2012) Architecture enhancements for non-3GPP accesses, TS 23.402 v10.7.0 Release 10. 3rd Generation Partnership Project (3GPP)

[25] 3GPP (March 2012) Access to the 3GPP Evolved Packet Core (EPC) via non-3GPP access networks; Stage 3, TS 24.302 v10.7.0 Release 10. 3rd Generation Partnership Project (3GPP)

[26] Vidal I, Soto I, Calderon M, Garcia-Reinoso J, Sandonis V (July 2013) Transparent Network-Assisted Flow Mobility for Multimedia Applications in IMS environments. IEEE Commun Mag 57 (7):97-105

[27] Sparks R (April 2003) The Session Initiation Protocol (SIP) Refer Method, RFC 3515. Internet Engineering Task Force

[28] Rosenberg J (Oct. 2009) Obtaining and Using Globally Routable User Agent URIs (GRUUs) in the Session Initiation Protocol (SIP), RFC 5627. Internet Engineering Task Force

[29] Mahy R, Biggs B, Dean R (Sep. 2004) The Session Initiation Protocol (SIP) "Replaces" Header, RFC 3891. Internet Engineering Task Force

[30] Lister D, Dehghan S, Owen R, Jones P (Mar 2000) UMTS capacity and planning issues. In First International Conference on 3G Mobile Communication Technologies, pp 218-223 